\title{
La acupuntura reduce la disnea de esfuerzo en pacientes con EPOC
}

\author{
Acupuncture reduces dyspnea on exertion in patients with COPD
}

Suzuki y col. Arch Int Med. 2012;172(11):878-886.

\section{Objetivo}

Determinar si la acupuntura es superior a la acupuntura placebo para mejorar la disnea de esfuerzo en pacientes con enfermedad pulmonar obstructiva crónica (EPOC) que se encuentran bajo tratamiento estándar.

\section{Diseño}

Ensayo clínico aleatorizado, simple ciego.

Lugar

4 hospitales de la región de Kansai, Japón.

\section{Pacientes}

Participaron 68 pacientes ambulatorios con diagnostico de EPOC grado II, III o IV (de la clasificación GOLD); clínicamente estables (sin antecedente de infecciones o exacerbaciones de síntomas respiratorios ni cambios en la medicación en los tres meses anteriores al estudio, y sin signos de edema); que pudieran caminar sin requerir asistencia; y que no hubieran realizado rehabilitación pulmonar en los últimos seis meses. Completaron el estudio 32 de 34 y 30 de 32 pacientes asignados al grupo intervención y placebo, respectivamente.

\section{Intervención}

Los pacientes se aleatorizaron a acupuntura real una vez a la semana durante 12 semanas, realizando una selección de puntos estandarizada de acuerdo a lo descripto en la literatura o a acupuntura placebo (simulado) mediante un dispositivo que contiene a la aguja dentro de un tubo que va adherido a la piel, las agujas placebos son romas y al aplicarlas parece que penetran en la piel pero en realidad se pliegan telescopicamente, mientras que la aguja real es afilada e ingresa en la piel unos
5 a $25 \mathrm{~mm}$.

Los pacientes, evaluadores y estadísticos estaban cegados a la intervención, no así los acupuntores.

El resultado primario (medido antes y después de 12 semanas de tratamiento) fue el cambio en la escala de Borg modificada luego de la prueba de caminata de seis minutos, cuyo puntaje va de cero (disnea nula) a diez (disnea máxima).

\section{Resultados principales}

Luego de 12 semanas de tratamiento, el puntaje de Borg sólo mejoró en el grupo intervención (Tabla 1). Los pacientes que recibieron acupuntura real también mejoraron la distancia caminada a los seis minutos, la saturación de oxígeno durante la caminata y en una escala de calidad de vida, mientras que los cambios en el VEF1 no alcanzaron significación estadística.

Tabla 1. Media ( \pm Desvío estándar) en la escala de Borg a los seis minutos de caminata.

\begin{tabular}{l|c|c}
\hline $\begin{array}{c}\text { Escala de disnea de Borg } \\
\text { (0 nula a 10 máxima) }\end{array}$ & $\begin{array}{c}\text { Grupo Acupuntura } \\
(\mathbf{n = 3 0})\end{array}$ & $\begin{array}{c}\text { Grupo Placebo } \\
(\mathbf{n = 3 2})\end{array}$ \\
\hline Puntaje basal & $5,5( \pm 2,8)$ & $4,2( \pm 2,7)$ \\
\hline Puntaje final & $1,9( \pm 1,5)$ & $4,6( \pm 2,8)$ \\
\hline Cambio (basal - final) & $-3,6( \pm 1,9)$ & $0,4( \pm 1,2)$ \\
\hline \multicolumn{2}{|c|}{$-3,6$ (IC95\% de $-4,3$ a $-2,9)$} \\
\hline
\end{tabular}

\section{Conclusión}

La acupuntura podría ser eficaz como tratamiento adyuvante para reducir la disnea de esfuerzo en pacientes con EPOC.

Fuente de financiamiento: Grants in-Aid for scientific research from the Japan Society of Acupuncture and Moxibustion (JSAM)

\section{Comentario}

Una limitación del estudio es la ausencia de análisis por intención de tratar*. El $12 \%$ y $6 \%$ de pérdidas en el grupo intervención y control respectivamente, podría haber modificado los resultados. Otro inconveniente, y quizá una de las principales limitaciones de la acupuntura en el desarrollo de ensayos clínicos, es la ausencia de doble ciego. Sin embargo, en el presente trabajo pacientes, evaluadores y estadísticos estuvieron cegados a la intervención y también se verificaron mejorías en parámetros objetivos como la saturación oxígeno y la distancia caminada a los seis minutos. Por otro lado, la estandarización de los puntos de acupuntura podría ser una fortaleza al aumentar la reproducibilidad de la técnica. El estudio mostró al igual que en estudios previos ${ }^{1,2}$ una mejoría en la disnea de esfuerzo comparable en magnitud a otras intervenciones como rehabilitación o ejercicio ${ }^{3,4}$, pero se requiere un seguimiento más prolongado para evaluar la duración del efecto, la adherencia a un tratamiento semanal y evaluar también la costo-efectividad de la intervención.

\section{Conclusiones del comentador}

Es válido considerar a la acupuntura como tratamiento complementario en los pacientes con EPOC. El desarrollo y la validación de un instrumento que sea útil para cegar al acupuntor, al igual que al paciente, sería un gran avance para realizar ensayos con menor riesgo de sesgo.

Plinio E. Gaglio [ Servicio de Medicina Familiar y Comunitaria del Hospital Italiano de Buenos Aires ] plinio.gaglio@ hospitalitaliano.org.ar

Gaglio PE. La acupuntura reduce la disnea de esfuerzo en pacientes con EPOC. Evid Act Pract Ambul. Oct-Dic 2012;15(4):125. Comentado de: Suzuki M, Muro S, Ando Y, y col. A randomized, placebo-controlled trial of acupuncture in patients with chronic obstructive pulmonary disease (COPD): the COPD-acupuncture trial (CAT). Arch Int Med. 2012;172(11):878-886. PMID: 22905352.

\section{Referencias bibliográficas}

1. Jobst K, Chen JH, McPherson K, y col. Controlled trial of acupuncture for disabling breathlessness. Lancet.1986;2(8521-22):1416-19.

2. Lewith GT, Prescott P, Davis CL. Can a standardized acupuncture technique palliate disabling breathlessness? A single-blind, placebo-controlled crossover study. Chest. 2004;125(5):1783-90.

3. Ries AL. Minimally clinically important difference for the UCSD Shortness of Breath Questionnaire, Borg scale, and visual analog scale. COPD. 2005;2(1):105-110.

4. Sciurba F, Criner GJ, Lee SM, y col. National Emphysema Treatment Trial Research Group. Six-minute walk distance in chronic obstructive pulmonary disease: reproducibil-

ity and effect of walking course layout and length. Am J Respir Crit Care Med. 2003;167(11):1522-27. 